\title{
SFRP5 mediates downregulation of the wnt5a/caveolin-1/JNK signaling pathway
}

\author{
Weihua Liu1,2,*, Yuqiang Ji3,*, Haiping Chu², Mo Wang ${ }^{4}$, Bin Yang ${ }^{4}$ and Chunyan Yin ${ }^{1}$ \\ 'Department of Pediatrics, The Second Affiliated Hospital of Xi'an Jiaotong University, Xi'an, Shaanxi, China \\ 2Department of Pediatrics, First Hospital of Xi'an, Xi'an, Shaanxi, China \\ ${ }^{3}$ Department of Cardiovascular Medicine, First Hospital of Xi'an, Xi'an, Shaanxi, China \\ ${ }^{4}$ Department of Pediatrics, Maternal and Child Health Hospital of Chang'an District, Xi'an, Shaanxi, China
}

Correspondence should be addressed to C Yin: xiaoyanfenggroup@sina.com

*(W Liu and Y Ji contributed equally to this work)

\begin{abstract}
This study investigated the effects of Wnt5a/caveolin/JNK signaling pathway and SFRP5 protein on ox-LDL-induced apoptosis of HUVEC cells. The difference of serological indexes between healthy average weight and obese children and the expression of Wnt $5 \mathrm{a}$ and SFRP5 was detected by clinical examination, and the correlation between serum SFRP5, Wnt 5a and the vascular endothelial injury was detected. HUVEC cells were induced by ox-LDL to construct an endothelial injury model, siRNA-transfected cells were used to construct downregulated SFRP5 and Wnt 5a expression groups, and recombination methods were used to construct upregulated Wnt5a and SFRP5 expression groups. The expression of Wnt 5 a, caveolin-1, JNK and apoptosis-related proteins under different treatments were detected by the Western blot method, and apoptosis was detected by flow cytometry. Serological results showed that the level of Sfrp5 in obese children was significantly lower than that in healthy children, and the level of Wnt5a was significantly higher than that in healthy children. Moreover, Ln Sfrp5 was significantly negatively correlated with Ang- 2 in blood circulation, ICAM-1 and E-selectin selectin, but not with VCAM-1. When Wnt5a was upregulated, the expression of caveolin-1 and JNK increased significantly, Bcl-2 decreased significantly, and the apoptotic rate increased significantly. Nevertheless, when Sfrp5 expression was upregulated, the result was the opposite. SFRP5 and Wnt5a are involved in the vascular endothelial injury. Wnt5a can promote apoptosis of HUVEC cells through Wnt5a/JNK/ Caveolin-1 pathway, while SFRP5 can inhibit apoptosis by interfering with this pathway.
\end{abstract}

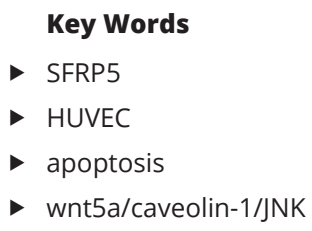

Journal of Endocrinology (2020) 247, 263-272

\section{Introduction}

Obesity is a chronic metabolic disorder caused by many factors, which has become the most common nutritional disorder in modern society. Obesity is a predisposing factor for metabolic diseases such as type 2 diabetes, often associated with low inflammation of adipose tissue (Akash et al. 2012). The imbalance of proinflammatory cytokines and anti-inflammatory cytokines in adipose tissue is an essential cause of obesity (Loos et al. 2008). Obesity is often accompanied by the increase of Inflammatory cytokines and the decrease of adiponectin, which has anti-infection and improves insulin resistance, leading to insulin resistance and macrophage infiltration of fat 
tissue (Arita 2012). Atherosclerosis is a complex process of cardiovascular, inflammatory lesions. It is characterized by the deposition of subintimal lipids in some parts of the artery, accompanied by the proliferation of smooth muscle cells and fibrous matrix components, which gradually develop into atherosclerotic plaques. Many studies have confirmed that excessive adipose tissue deposition is closely related to the formation of atherosclerosis, and obesity is an independent risk factor for the development of atherosclerosis (Danaei et al. 2009, Bilkovski et al. 2011).

Many signal molecules of the Wnt signaling pathway play an essential role in metabolism regulation and tissue inflammation. Wnt/ $\beta$-Catenin and $\mathrm{Wnt} / \mathrm{Ca}^{2+}$ maintain proinflammatory and anti-inflammatory factors (Schulte et al. 2012, Qin et al. 2014, Khosravi et al. 2014, Ma et al. 2015). Ian Ackers et al. also found that the Wnt 5a signaling pathway has a direct role in the pathogenesis of atherosclerosis, is significantly expressed in advanced atherosclerosis, and rWnt 5a significantly increases lipid accumulation in THP-1 macrophages (Ackers et al. 2018). JNK signaling pathway mediates the release of apoptotic factors from mitochondria, phosphorylates BCL, promotes the expression of apoptotic family members (such as Bak and bid), and further induces the expression of proinflammatory cytokines such as tumor necrosis factor alpha (TNF-a), interleukin-1 (IL-1) and interleukin-6 (IL-6) (Liu \& Lin 2005). Secretory frizzled-related proteins (SFRPS) are a class of Wnt signal pathway inhibitory proteins. SFRP5 is a newly identified anti-inflammatory fat factor that can competitively bind Wnt signal pathway extracellular ligand Wnt5a protein, inhibit the Wnt signal pathway, and alleviate chronic inflammatory state. Wang Di elaborated on the anti-inflammatory effects of SFRP5 in obesity, type II diabetes and coronary heart disease in his research, and considered SFRP5 as the most promising target for treating obesity, coronary heart disease and type II diabetes (Wang et al. 2020). SFRP5 can improve metabolism disorder by inhibiting inflammatory cell activation in adipose tissue, and Wnt/JNK may be the critical signal pathway in this process (Ouchi et al. 2010). Caveolin-1 is the structural protein of caveolae on the cell membrane. Caveolin-1 is expressed in many kinds of cells, mainly in adipocytes, smooth muscle cells, endothelial cells, epithelial cells, fibroblasts and other end differentiated cells (Rothberg et al. 1992). Caveolin-1 can be expressed in neutrophils, macrophages, lymphocytes and dendritic cells, which indicates that caveolin-1 plays a vital role in the physiological effects of inflammatory cells and participates in the regulation of signal transduction on the surface of leukocytes (Hu et al. 2008).
Endothelial cell damage or dysfunction can lead to increased vascular wall permeability, aggravated inflammatory response, and trigger thrombosis. Endothelial cell apoptosis is the main form of endothelial injury and plays an important role in atherosclerosis occurrence and development. Wnt5a can activate caveolin-1 to participate in the formation of atherosclerosis in oxidative low-density lipoprotein (LDL) induced vascular smooth muscle injury (Qin et al. 2014). Caveolin-1a activates JNK against Bcl-2, leading to apoptosis. SFRP5 can inhibit the Wnt signaling pathway and alleviate the chronic inflammatory state (Nakamura et al. 2014). Firstly, the expression differences of Wnt $5 \mathrm{a}$ and SFRP5 in the serum of healthy weight children and obese children were analyzed clinically, and the correlation between Wnt 5a and SFRP5 and the vascular endothelial injury was analyzed. Finally, ox-LDL was used to induce HUVEC to establish an endothelial injury cell model, to explore the regulation of the Wnt $5 \mathrm{a} /$ caveolin-1/ JNK signaling pathway on the apoptosis of HUVEC cells treated with ox-LDL, and the inhibitory effect of SFRP5 on signaling pathway. In atherosclerosis, this signaling pathway has not been thoroughly studied and reported. Therefore, this study can provide a theoretical basis for the clinical study of atherosclerosis.

\section{Materials and methods}

\section{Study population}

The study was approved by the Ethics Committee of the Second Affiliated Hospital of Xi'an Jiaotong University, and written informed consent was obtained from all participants and their families. There were 120 subjects

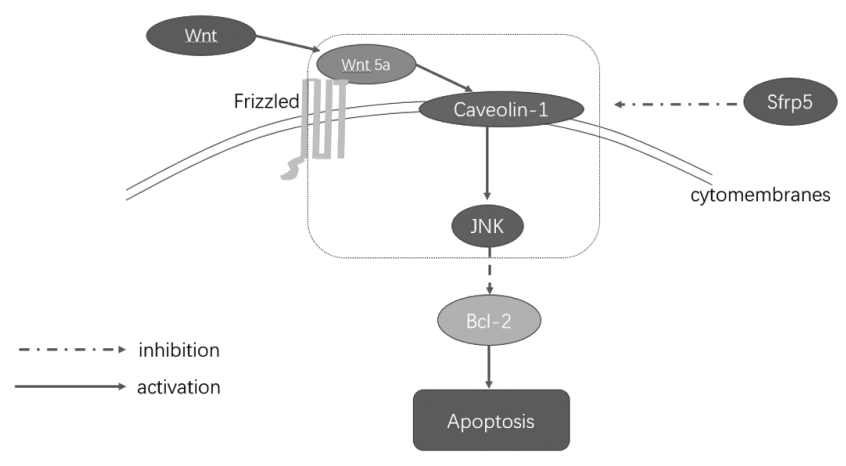

Sfrp5/Wnt5a/Caveolin-1/JNK Cell signaling pathway

Figure 6

Sfrp5/Wnt5a/caveolin-1/JNK signal mechanism diagram. 
in this experiment, including $n=80$ obese children and $n=40$ normal-weight children. Inclusion criteria: (1) Age between 8 and 12; (2) clear clinical background, no liver, and thyroid-related diseases; (3) no congenital diseases and genetic defects; Exclusion criteria: (1) acute or chronic infectious diseases; (2) congenital diseases; (3) patients with untreated thyroid disease; (4) growth hormone deficient patients.

\section{Serological testing}

Systolic blood pressure (SBP), diastolic blood pressure (DBP), BMI, SDS-BMI, waist-hip ratio, waist-height ratio (WHtR), SDS-SBP, SDS-DBP, homeostasis model insulin resistance index (HOMA-IR), triglyceride, LDL-cholesterol, fasting plasma glucose (FPG) and total cholesterol (TC), HDL-cholesterol, fasting insulin were measured in all children; all the above tests were completed in the fasting state.

\section{Detection of correlation between serum SFRP5 or Wnt5a, metabolism and vascular endothelial damage index}

Cell adhesion molecules, including E-selectin, intercellular cell adhesion molecule-1 (ICAM-1) and vascular cell adhesion molecule-1 (VCAM-1), were selected as vascular endothelial damage indexes to detect the correlation between SFRP5 or Wnt5a and anthropometric indexes, metabolic indexes and vascular endothelial damage.

\section{Cell culture and treatment}

The cell was cultured with RPMI 1640 of $20 \%$ fetal bovine serum. Human Umbilical Vein Endothelial Cells (HUVEC cells cultured to the third-generation were used as control group and the ox-LDL treatment group. HUVEC cells were treated with 25, 50, and $100 \mu \mathrm{g} / \mathrm{mL}$ ox LDL for $48 \mathrm{~h}$ to establish the endothelial cell injury model.

\section{Detection of cell proliferation by CCK-8 assay}

The cells to be tested were treated according to different experimental conditions and incubated in the incubator for 0,24 and $48 \mathrm{~h}$. Ten microliters CCK-8 (Genview, Beijing, China) solution was added to each well, and incubation was continued in the incubator. A microplate reader read the OD value of $450 \mathrm{~nm}$ wavelength.

\section{Detection of apoptosis by flow cytometry}

In each group, cells were inoculated into six-well cell culture plate, and blank control and positive control holes were set. Annexin V-FITC and PI (BD Biosciences, CA, USA, Catalog No. 550911) were used for staining, ice bath and dark place were used for flow cytometry (FACScalibur, BD Biosciences) to detect apoptosis, and the data were quantified by FCS Express software(BD CellQuest Pro); annexin V-FITC was green fluorescence and PI was red fluorescence.

\section{Detection of protein expression by Western blotting}

Each group's total protein was extracted with RIPA Lysis Buffer (RIPA Lysis Buffer, CoWin Biosciences, Beijing, China, Catalog No. CW2334S) mammalian protein extraction reagent. The total proteins concentrations were quantified using BCA Protein Assay kit (Micro BCA Protein Assay Kit, CoWin Biosciences, Catalog No. CW2011S). The Western blotting system was established using the Bio-Rad Bis-Tris Gel system (Bio-Rad Laboratories). After SDS-PAGE electrophoresis, the total protein extract was transferred to PVDF membrane (Millipore); All primary antibodies were purchased from Santa Cruz Biotechnology, Wnt 5a, SFRP5, caveolin-1, JNK, Bcl-2 (1:250), polyclonal antibody of $\beta$-actin (1:1000), dilution ratio of the second antibody was 1:1000; Fusion software was used to analyze the development results with $\beta$-actin as internal reference; Quantity one analysis software (Bio-Rad Laboratories) was used to analyze the development results.

\section{siRNA cell transfection}

Try to select the target with the highest interference efficiency for multiple pairs of siRNA fragments. siRNA gene sequence and synthesis provided by Shanghai Genechem company (Shanghai, China). Take $0.67 \mu \mathrm{g}$ (50 pmol) of siRNA, add a certain amount of serum-free diluent; take $1 \mu \mathrm{L}$ of EntransterTM-R4000 (Shanghai, China), then add $24 \mu \mathrm{L}$ of serum-free diluent; fully mix the EntransterTM-R4000 diluent and RNA diluent; drop $50 \mu \mathrm{L}$ of the transfection complex onto the cell with $0.45 \mathrm{~mL}$ of full culture medium (can contain 10\% serum and antibiotics); observe the cell state $6 \mathrm{~h}$ after transfection.

\section{Statistical analysis}

All experiments in this research were performed in triplicate. Values were expressed as mean \pm S.D. Statistical analysis was performed using SPSS 21.0; Statistical analysis 
was performed using Student's $t$-test. $P$ values among the groups were calculated using one-way ANOVA. $P<0.05$ was considered to be statistically significant.

\section{Results}

\section{Differences in clinical characteristics and Sfrp5 levels between the two groups}

There was no significant difference in age and gender between the two groups. BMI, SDS-BMI, waist to hip ratio, WHtR, systolic pressure, diastolic pressure, systolic pressure (SBP), diastolic blood pressure (DBP), homeostasis model assessment of insulin resistance (HOMA-IR), triglyceride and LDL cholesterol levels in obese children $(P<0.05)$. There was no significant difference in fasting plasma glucose (FPG), total serum cholesterol (TC), and high-density lipoprotein (HDL) cholesterol between the obese group and the control group. The level of SFRP5 in obese children was significantly lower than that in healthy children, and the level of Wnt5a was significantly higher than that in healthy children. The concentrations of ICAM-1, VCAM-1, Ang-2, and E-selectin in obese children were significantly higher than those in normalweight children $(P<0.05)$. The clinical characteristics of the subjects are shown in Table 1 .

\section{Correlation between serum SFRP5 or Wnt5a and metabolic and vascular endothelial damage indexes}

Ln SFRP5 was negatively correlated with Ang-2, ICAM-1, and E-selectin $(P<0.05)$, but not with VCAM-1. There was a significant positive correlation between Ln Wnt 5a and Ang-2, ICAM-1, and E-selectin in blood circulation $(P<0.05)$. It is suggested that SFRP5 or Wnt5a may be involved in vascular endothelial dysfunction. Detailed experimental results are shown in Table 2.

\section{Morphological results of HUVEC}

The results of HUVEC cells treated with different methods are shown in Fig. 1A. With the increase of ox LDL content and incubation time, the cell surface bulge gradually increased. The distribution became more and more irregular, accompanied by depression or cavity; the cell surface structure of the control group changed little with the increase of incubation time. Printed in Great Britain
Table 1 Clinical and laboratory characteristics of study population $(\bar{x} \pm s)$.

\begin{tabular}{|c|c|c|c|}
\hline Characteristics & Lean (40) & Obese $(80)$ & $\boldsymbol{P}$ \\
\hline pAge (year) & $11.26 \pm 2.05$ & $12.23 \pm 2.62$ & 0.091 \\
\hline $\mathrm{BMI}\left(\mathrm{kg} / \mathrm{m}^{2}\right)$ & $18(15,21)$ & $27(24,30)$ & $<0.01$ \\
\hline SDS-BMI & $2.62 \pm 0.41$ & $2.89 \pm 0.35$ & $<0.01$ \\
\hline WHtR & $0.42 \pm 0.21$ & $0.65 \pm 0.26$ & 0.023 \\
\hline WHR & $0.82 \pm 0.06$ & $1.04 \pm 0.13$ & 0.036 \\
\hline SDS-SBP & $1.43 \pm 0.79$ & $1.82 \pm 0.83$ & 0.025 \\
\hline SDS-DBP & $0.97 \pm 0.38$ & $1.17 \pm 0.62$ & 0.042 \\
\hline FPG (mmol/L) & $4.62 \pm 0.89$ & $5.01 \pm 0.54$ & 0.12 \\
\hline HOMA-IR & $2.45(1.43,4.62)$ & $3.34(2.13,5.92)$ & 0.034 \\
\hline $\begin{array}{l}\text { Fasting insulin } \\
(\mathrm{mlU} / \mathrm{L})\end{array}$ & $1.72 \pm 0.26$ & $2.12 \pm 0.14$ & 0.014 \\
\hline $\mathrm{TC}(\mathrm{mmol} / \mathrm{L})$ & $3.62 \pm 0.56$ & $3.92 \pm 0.64$ & 0.078 \\
\hline TG $(\mathrm{mmol} / \mathrm{L})$ & $1.18 \pm 0.35$ & $1.62 \pm 0.58$ & 0.016 \\
\hline HDL-C (mmol/L) & $1.36 \pm 0.35$ & $1.38 \pm 0.43$ & 0.161 \\
\hline LDL-C (mmol/L) & $2.58 \pm 0.90$ & $2.81 \pm 1.03$ & 0.031 \\
\hline Sfrp5 (ng/mL) & $7.89(4.28,9.04)$ & $3.56(3.10,5.82)$ & $<0.01$ \\
\hline Wnt5a (ng/mL) & $1.12(0.92,1.92)$ & $1.68(1.11,2.21)$ & $<0.01$ \\
\hline ICAM-1 ( $\mu \mathrm{g} / \mathrm{mL})$ & $5.81 \pm 1.52$ & $12.43 \pm 2.01$ & $<0.01$ \\
\hline VCAM-1 $(\mu \mathrm{g} / \mathrm{mL})$ & $142.36 \pm 18.62$ & $221.43 \pm 35.42$ & $<0.01$ \\
\hline Ang-2 (pg/mL) & $92.35 \pm 12.30$ & $131.45 \pm 18.65$ & $<0.01$ \\
\hline $\begin{array}{l}\text { E-selectin } \\
(\mathrm{ng} / \mathrm{mL})\end{array}$ & $17.32 \pm 4.65$ & $35.64 \pm 8.64$ & $<0.01$ \\
\hline
\end{tabular}

The proliferation of HUVEC cells treated with different concentrations of ox-LDL

There was no difference in the proliferation rate of cells treated with different ox-LDL concentrations at $0 \mathrm{~h}$. After $24 \mathrm{~h}$ of treatment, the cell proliferation rate gradually decreased with the increase of ox-LDL concentration. Compared with the control group, when the concentration of ox-LDL was 50 and $100 \mu \mathrm{g} / \mathrm{mL}$, the cell proliferation rate significantly decreased $(P<0.05)$. After $48 \mathrm{~h}$, when the concentration of ox-LDL was 50 and $100 \mu \mathrm{g} / \mathrm{mL}$, the cell proliferation rate decreased significantly compared with the control group $(P<0.01)$ (Fig. 1B and C).

\section{Analysis of apoptosis and expression of related proteins in HUVEC cells treated with different concentrations of ox-LDL}

It can be seen that $48 \mathrm{~h}$ after treatment with different ox LDL concentrations, the apoptosis of cells is different (Fig. $2 \mathrm{~A}$ and $\mathrm{B})$. Compared with the control group, the apoptosis rate and necrosis rate increased with the concentration increase. When the concentration of ox-LDL was more than $50 \mu \mathrm{g} / \mathrm{mL}$, the apoptosis rate and necrosis rate of the HUVEC cells were significantly different from the control group $(P<0.01)$. Forty-eight $\mathrm{h}$ after treatment with $25 \mu \mathrm{g} / \mathrm{mL}$ ox-LDL, compared with the control group, 
Table 2 Simple linear regression analysis using SFRP5 and Wnt5a as dependent.

\begin{tabular}{|c|c|c|c|c|}
\hline \multirow[b]{2}{*}{ Variables } & \multicolumn{2}{|c|}{ Ln Sfrp5 (ng/mL) } & \multicolumn{2}{|c|}{ Ln Wnt5a (ng/mL) } \\
\hline & $\beta(r)$ & $P$ & $\beta(r)$ & $P$ \\
\hline \multicolumn{5}{|c|}{ Anthropometric parameters } \\
\hline BMI $\left(\mathrm{kg} / \mathrm{m}^{2}\right)$ & -0.543 & 0.000 & 0.502 & 0.000 \\
\hline SDS-BMI & -0.581 & 0.000 & 0.563 & 0.000 \\
\hline WC (cm) & -0.432 & 0.000 & 0.352 & 0.000 \\
\hline WHR & -0.465 & 0.000 & 0.423 & 0.000 \\
\hline WHtR & -0.385 & 0.000 & 0.352 & 0.000 \\
\hline \multicolumn{5}{|l|}{ Metabolic parameters } \\
\hline FPG (mmol/L) & -0.178 & 0.096 & 0.145 & 0.235 \\
\hline Insulin (pmol/L) & -0.192 & 0.081 & 0.162 & 0.219 \\
\hline LnHOMA-IR & -0.204 & 0.072 & 0.181 & 0.118 \\
\hline LDL-C (mmol/L) & -0.185 & 0.089 & 0.121 & 0.345 \\
\hline HDL-C (mmol/L) & -0.196 & 0.084 & 0.048 & 0.720 \\
\hline $\operatorname{LnTG}(\mathrm{mmol} / \mathrm{L})$ & 0.023 & 0.860 & 0.052 & 0.716 \\
\hline TC (mmol/L) & 0.115 & 0.090 & 0.188 & 0.395 \\
\hline \multicolumn{5}{|l|}{ Endothelialmarkers } \\
\hline SDS-SBP & -0.351 & 0.000 & 0.245 & 0.000 \\
\hline SDS-DBP & -0.437 & 0.000 & 0.262 & 0.000 \\
\hline Ang-2 (pg/mL) & -0.462 & 0.000 & 0.364 & 0.000 \\
\hline VCAM-1 $(\mu \mathrm{g} / \mathrm{mL})$ & -0.121 & 0.114 & 0.160 & 0.221 \\
\hline E-selectin (ng/mL) & -0.358 & 0.000 & 0.423 & 0.000 \\
\hline ICAM-1 $(\mu \mathrm{g} / \mathrm{mL})$ & -0.442 & 0.000 & 0.336 & 0.000 \\
\hline
\end{tabular}

the apoptosis rate was significant $(P<0.05)$, but the necrosis rate had no significant difference.

With the different concentrations of ox LDL, different proteins showed different changes (Fig. 2C and D).
With the increase of ox LDL concentration, the expression of Wnt 5a, caveolin-1, JNK increased gradually, and the expression of SFRP5 and Bcl-2 decreased. When the ox-LDL concentration was $25 \mu \mathrm{g} / \mathrm{mL}$, the expression

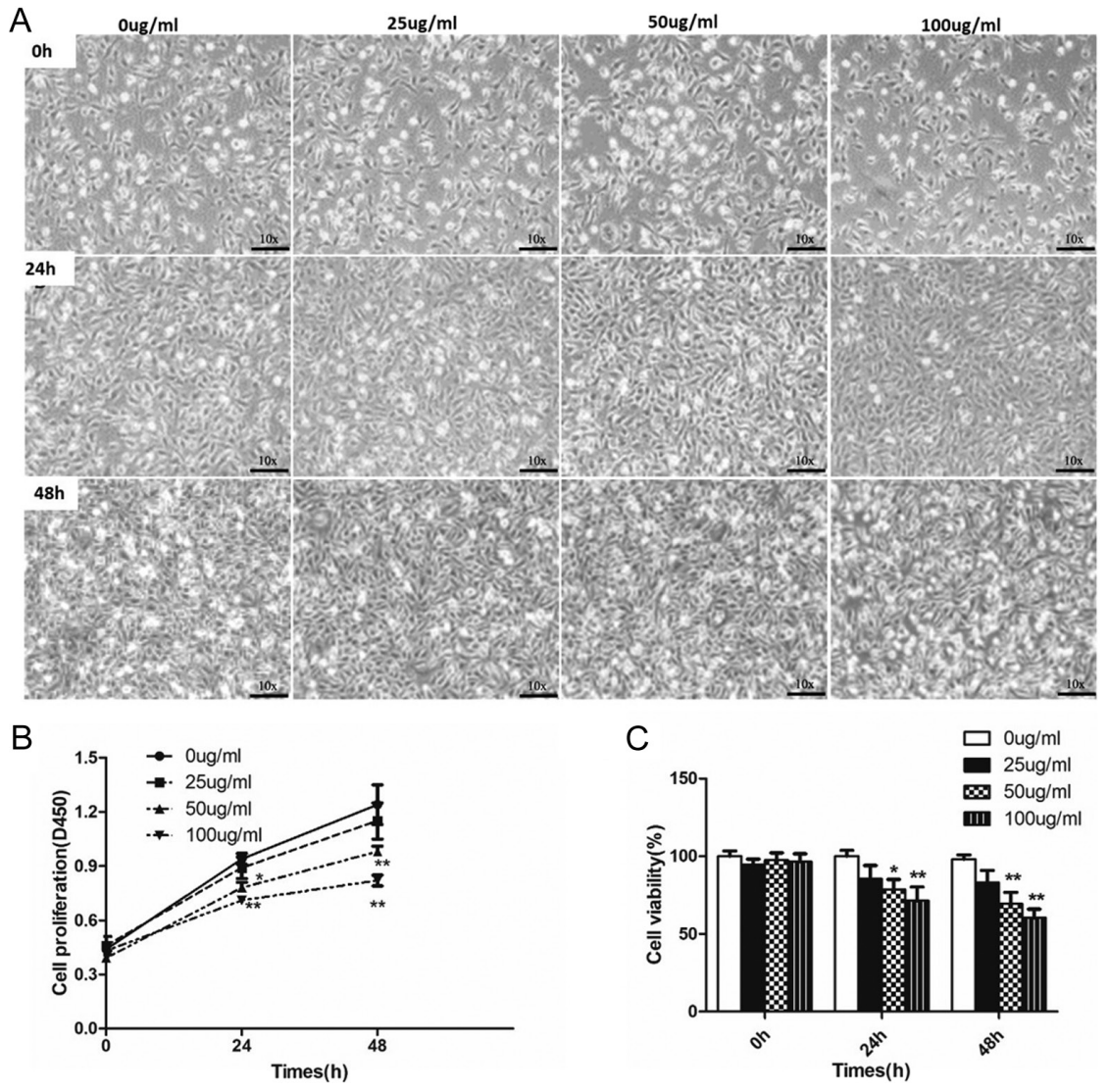

\section{Figure 1}

Cell morphology and cell proliferation of HUVEC cells treated with different concentrations of ox-LDL over time. (A) Group A represents the cell change map of $0 \mathrm{~h}$ treatment time, group $\mathrm{B}$ represents the cell change map of HUVEC after $24 \mathrm{~h}$ treatment, and group C represents the cell change map after $48 \mathrm{~h}$ treatment; all groups represent the concentration of ox LDL from left to right, which are $0,25,50$, and $100 \mu \mathrm{g} / \mathrm{mL}$ respectively. With the increase of ox-LDL content and incubation time, the cell surface bulge gradually increased, and the distribution became more and more irregular, accompanied by depression or cavity; the cell surface structure of the control group changed little with the increase of incubation time. (B) Detection of OD450 value of cell proliferation at different concentration and different time by CCK-8. (C) CCK-8 detection of cell proliferation rate at different concentrations and different time. ${ }^{*} P<0.05, * * P<0.01$. 

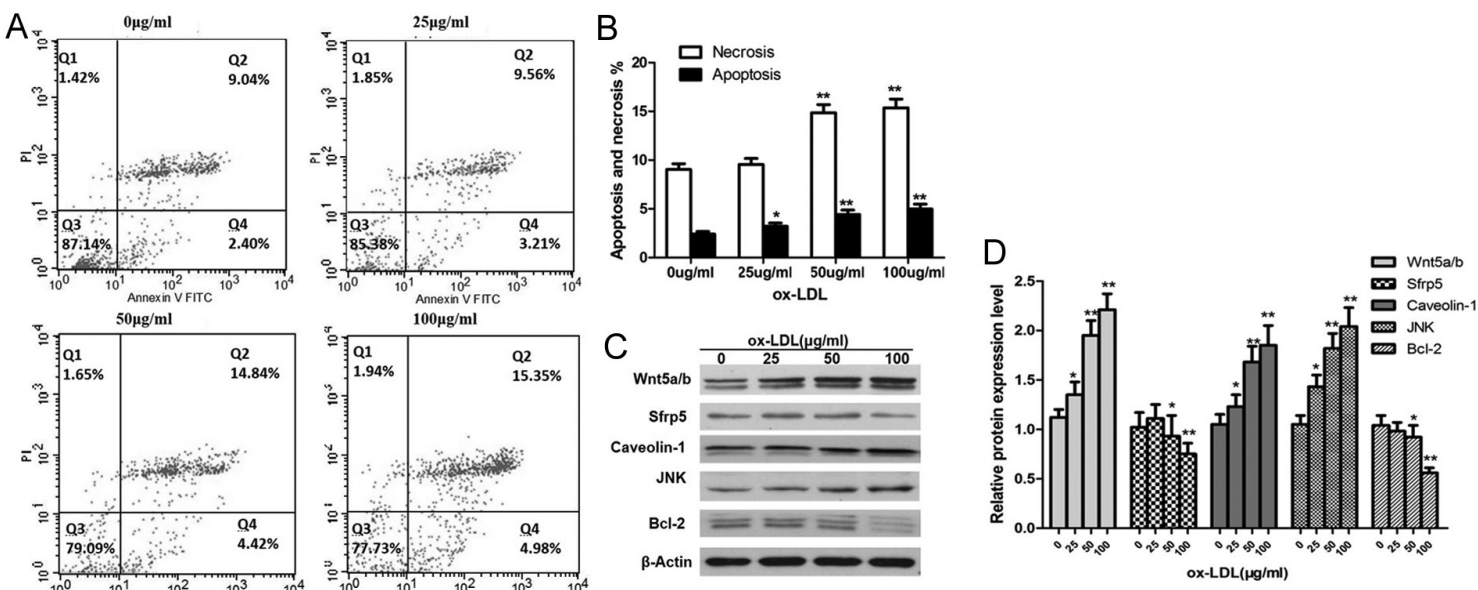

\section{Figure 2}

Analysis of apoptosis and expression of related proteins in HUVEC cells treated with different concentrations of ox-LDL. (A) Flow cytometry to detect apoptosis in each group. The lower-left quadrant of each image represents normal cells, and PI and AV staining were negative (AV-/pl-). The lower right quadrant represents apoptotic cells. AV positive and PI negative ( $\mathrm{AV}+/ \mathrm{PI}-$ ). The upper right quadrant shows necrotic cells and positive for AV and PI staining (AV+/PI+). (B) Comparison of cell necrosis rate and apoptosis rate in each group. When the concentration of ox-LDL is $\mathrm{more}$ than $50 \mu \mathrm{g} / \mathrm{mL}$, the difference between apoptosis rate and cell necrosis rate is very significant compared with the control group. When the concentration of ox LDL is $25 \mu \mathrm{g} / \mathrm{mL}$, the difference between apoptosis rate and necrosis rate is significant compared with the control group. (C and D) The expression of Wnt5a, sfrp5a, caveolin-1, JNK and Bcl-2 were detected by Western blot. With the increase of ox LDL concentration, Wnt5a, caveolin-1, JNK expression increased, Sfrp5 and bcl-2 expression decreased. $* P<0.05, * * P<0.01$.

of Wnt 5a, caveolin-1, JNK protein was significantly different from that of the control group $(P<0.05)$; when the ox-LDL concentration was 50 and $100 \mu \mathrm{g} / \mathrm{mL}$, the difference was extremely significant $(P<0.01)$. The protein expression of SFRP5 and $\mathrm{Bcl}-2$ was the opposite. When the concentration of ox-LDL was $25 \mu \mathrm{g} / \mathrm{mL}$, there was no significant difference with the control group $(P>0.05)$; when the concentration of ox-LDL was $50 \mu \mathrm{g} / \mathrm{mL}$, it was significantly different from the control $(P<0.05)$. When the concentration of ox-LDL was $100 \mu \mathrm{g} / \mathrm{mL}$, compared with the control group, the expression of SFRP5 and Bcl-2 proteins was very significant $(P<0.01)$.

\section{Wnt5a involved in the regulation of apoptosis of HUVEC cells treated with ox LDL}

HUVEC cells treated with $100 \mu \mathrm{g} / \mathrm{mL}$ ox-LDL for $48 \mathrm{~h}$. Wnt 5 a signal was induced by recombinant Wnt5a to set up the Wnt5a upregulation group, and the Wnt5a downregulation group was set up by Wnt5a siRNA. Flow cytometry to detect apoptosis in each group (Fig. 3A and $B)$. There was no significant difference between the control group and the scramble siRNA group $(P>0.05)$. Compared with the control group, the apoptosis rate of the Wnt $5 \mathrm{a}$ upregulated group increased (rWnt $5 \mathrm{a}$ ), but the necrosis rate decreased $(P<0.01)$. The difference is that in the Wnt 5a downregulation group (siWnt5a), the necrosis rate and apoptosis rate of HUVEC cells were significantly reduced. It is suggested that Wnt $5 \mathrm{a}$ is involved in the apoptosis of HUVEC cells treated with ox LDL, and the expression of Wnt 5 a is positively correlated with apoptosis.

\section{Caveolin-1 is a downstream molecule of Wnt5a involved in regulating HUVEC apoptosis induced by ox LDL}

HUVEC cells were treated with $100 \mu \mathrm{g} / \mathrm{mL}$ ox-LDL for $48 \mathrm{~h}$, then Wnt5a upregulated group (rWnt $5 \mathrm{a}$ group) and Wnt5a downregulated group (siWnt 5a group) were set. The control group was treated with $100 \mu \mathrm{g} / \mathrm{mL}$ ox-LDL alone. Using Western blotting to detect caveolin-1, JNK, Bcl-2 in rWnt 5a group, and siWnt 5a group cells, the results are shown in Fig. $3 \mathrm{C}$ and D. There was no significant difference in all proteins' expression between the control group (Single ox-LDL treatment group) and the Scramble siRNA group. The expression trend of JNK and Caveolin-1 was the same. Compared with the control group (Single ox-LDL treatment group), the protein expression of the rWnt5a group increased significantly $(P<0.01)$, while that of the siwnt5a group decreased significantly $(P<0.05)$. Compared with the control group (Single ox-LDL treatment group), the expression of Bcl-2 protein in the rwnt5a group was significantly lower $(P<0.05)$, but that in the siWnt5a group was significantly higher $(P<0.05)$. These results suggest that caveolin-1 is a downstream molecule of Wnt5a, which is involved in regulating HUVEC apoptosis by ox-LDL. 

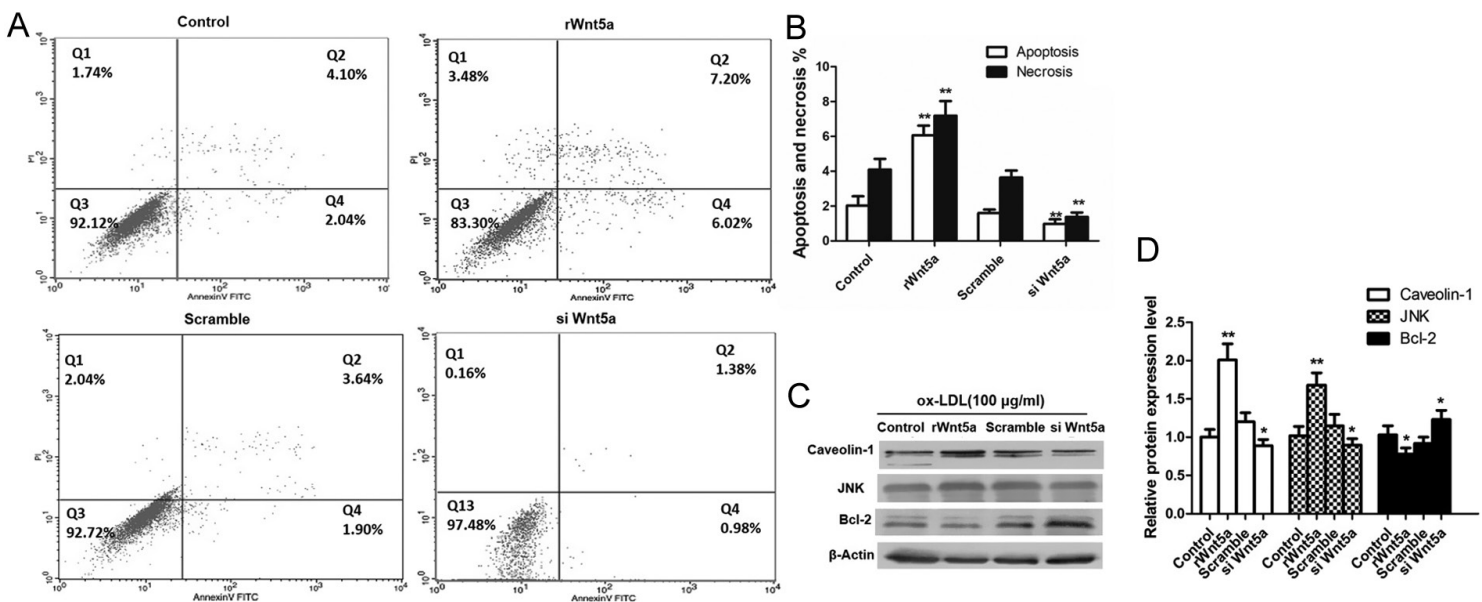

\section{Figure 3}

Effect of Wnt 5a on apoptosis and expression of downstream-related molecules in HUVEC cells. (A) Flow cytometry detection of cell apoptosis in different groups treated with $100 \mu \mathrm{g} / \mathrm{mL}$ ox-LDL HUVECs treated with $100 \mu \mathrm{g} / \mathrm{mL}$ ox-LDL for $48 \mathrm{~h}$ were set up with Wnt5a upregulation group and Wnt5a downregulation group, and apoptosis was detected by flow cytometry. The lower-left quadrant of each image represents normal cells, and PI and AV staining were negative ( $\mathrm{AV}-/ \mathrm{pl}-$ ). The lower right quadrant represents apoptotic cells. AV positive and $\mathrm{PI}$ negative (AV+/PI-). The upper right quadrant shows necrotic cells and positive for AV and PI staining (AV+/PI+). (B) Comparison of cell necrosis rate and apoptosis rate in each group. (C and D) Western blotting detection of caveolin-1, JNK, bcl-2 protein expression in each group. After HUVEC was treated with $100 \mu \mathrm{g} / \mathrm{mL}$ ox-LDL for $48 \mathrm{~h}$, the expression of caveolin-1 and JNK in rWnt5a group was higher than that in ox-LDL group, while that of caveolin-1 and JNK in siRNA Wnt5a group was lower than that in ox-LDL group. There was no significant difference in caveolin-1, JNK, bcl-2 between the ox-LDL groups and Scramble-siRNA group. $\star P<0.05, * \star P<0.01$.

\section{JNK is a downstream molecule of caveolin-1 involved in the regulation of HUVEC apoptosis treated by ox-LDL}

After treating HUVEC cells with $100 \mu \mathrm{g} / \mathrm{mL}$ ox-LDL for $48 \mathrm{~h}$, the control group and caveolin-1 downregulation group were set (siCaveolin-1 group). Caveolin-1 siRNA was used to regulate the expression of caveolin-1 down. The control group was treated with ox-LDL alone. The detection of apoptosis was done by flow cytometry (Fig. 4A and B).

There was no significant difference in apoptosis rates and necrosis between the control group and the Scramble group. Compared with the control group, apoptosis and necrosis rates were significantly reduced in the siCaveolin-1 group $(P<0.05)$.

Detection of JNK, Bcl-2 protein expression was done by WB method (Fig. 4C and D). After HUVEC was treated with $100 \mu \mathrm{g} / \mathrm{mL}$ ox-LDL for $48 \mathrm{~h}$, JNK expression decreased, and Bcl-2 expression increased significantly in sirna-caveolin-1 group $(P<0.05)$. The expression of JNK and Bcl-2 in the Scrabble siRNA group was not significantly different from that in the ox-LDL group.

\section{$\mathrm{Bcl}-2$ is a negative regulatory target of JNK and participates in HUVEC apoptosis regulation treated by ox LDL}

HUVEC cells were treated with $100 \mu \mathrm{g} / \mathrm{mL}$ ox-LDL for $48 \mathrm{~h}$, then the control group and JNK downregulation group (Sp600125 group) were set. Sp600125, a JNK inhibitor, was used to inhibit the expression of JNK. The control group was treated with ox-LDL alone. The expression of Bcl-2 was detected by Western blotting (Fig. 4E and F). Compared with the control group, the expression of Bcl-2 in the sp600125 group increased $(P<0.05)$.

\section{The role of SFRP5 in regulating the apoptosis of HUVEC cells treated with ox LDL}

After treatment of HUVEC cells with $100 \mu \mathrm{g} / \mathrm{mL}$ ox-LDL for $48 \mathrm{~h}$, the following groups were set up: rWnt5a group, rSFRP5+rWnt5a group, rWnt5a+siSFRP5 group, Scramble-siRNA + rWnt5a group, control group. Detection of apoptosis by flow cytometry (Fig. 5A and B). Compared with the control group, the apoptosis rate of rWnt5a group and scrabble siRNA + rWnt5a group increased significantly $(P<0.05)$; rWnt5a + Si SFRP5 group increased significantly $(P<0.01)$; but the apoptosis rate of rSFRP5 + rwnt5a group decreased significantly $(P<0.05)$.

The downstream protein expression was detected by WB method (Fig. 5C and D). Compared with the control group, the expression of Caveolin-1 and JNK in rSFRP5+ rwnt5a group decreased significantly, and Bcl-2 increased significantly $(P<0.01)$. It is suggested that SFRP5 can antagonize Wnt5a and inhibit apoptosis. 

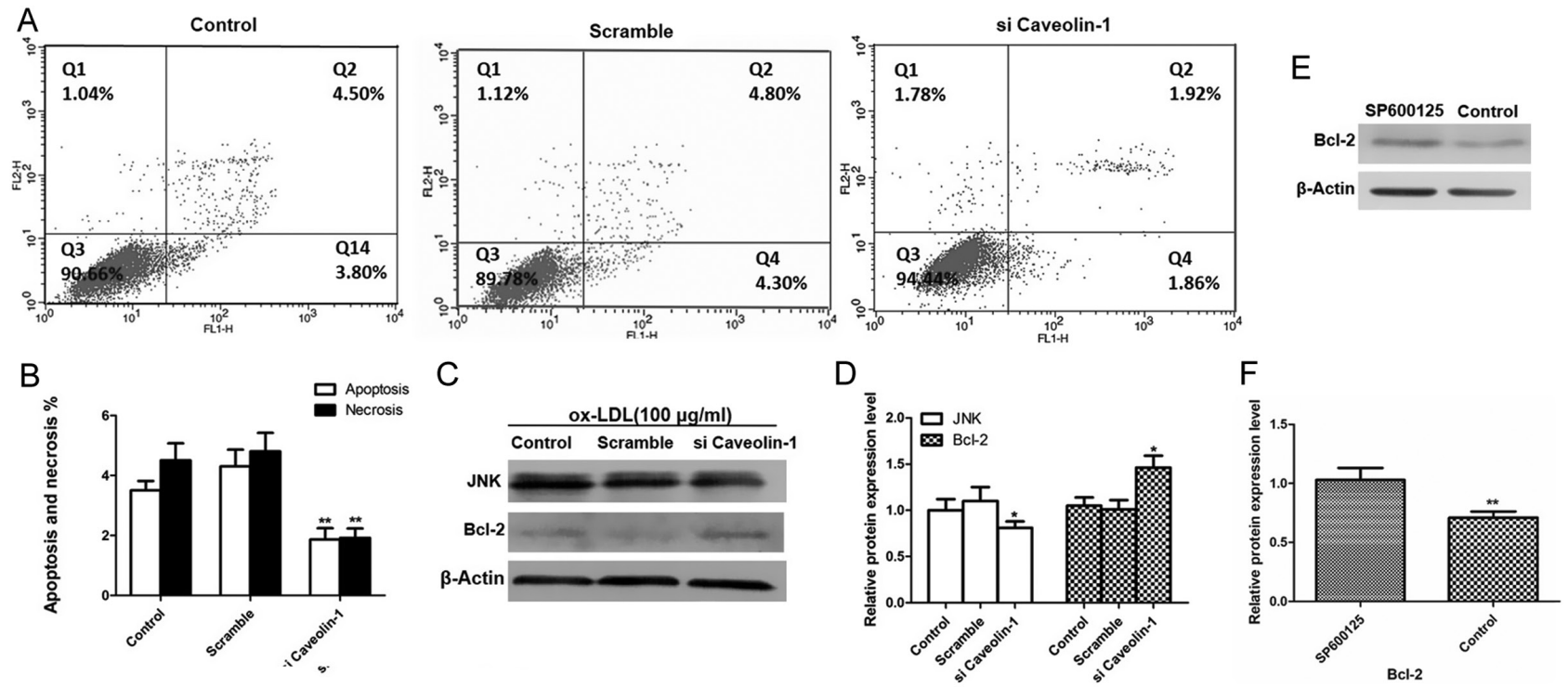

Figure 4

Effect of caveolin-1 on apoptosis and expression of downstream related molecules in HUVEC cells. (A) Flow cytometry detection of cell apoptosis in different groups treated with $100 \mu \mathrm{g} / \mathrm{mL}$ ox-LDL. HUVECs treated with $100 \mu \mathrm{g} / \mathrm{mL}$ ox-LDL for $48 \mathrm{~h}$ were set up with Wnt5a upregulation group and Wnt5a downregulation group, and apoptosis was detected by flow cytometry. The lower-left quadrant of each image represents normal cells, and PI and AV staining were negative (AV-/pl-). The lower right quadrant represents apoptotic cells. AV positive and PI negative (AV+/PI-). The upper right quadrant shows necrotic cells and positive for AV and PI staining (AV+/PI+). (B) Comparison of cell necrosis rate and apoptosis rate in each group. (C and D) Western detection of JNK and bcl-2 protein expression in each group. Compared with the control group, the expression of JNK protein decreased and $\mathrm{Bcl}-2$ increased in sicaveolin-1 group. (E and F) Effect of JNK on bcl-2 expression. Compared with the control group, the expression of Bcl-2 in sp600125 group increased. $* P<0.05, * * P<0.01$.

\section{Discussion}

Obesity is a high-risk factor of metabolic syndrome, diabetes, hypertension, hyperlipidemia, and arteriosclerotic disease (Danaei et al. 2009). Hotamisligil (2003) first proposed that obesity is a kind of systemic chronic low-grade inflammatory state induced by different inflammatory factors. It has been proved that adipose tissue's chronic inflammation is the key link between obesity and metabolic diseases. In recent years, with the in-depth study of obesity-induced chronic inflammation, its possible mechanism is gradually clarified, and the involved signaling pathway is also gradually reported.

Wnt signaling pathway is a highly conserved signal transduction pathway in vivo. It can be seen that the Wnt signaling pathway is involved and regulated in all stages of organisms from zygote division to adult development, from epidermal growth to visceral formation. The results show that the Wnt signaling pathway can inhibit the transformation of preadipocytes to adipocytes, thus inhibiting adipose tissue (Kikuchi et al. 2012, Okamura et al. 2014). Bilkovski et al. (2011) has also shown that macrophages could influence the adipogenesis of mesenchymal precursor cells through Wnt 5a in obese well as type II diabetic patients. It was found that the expression of TNF- $\alpha$ and GSK3 and $\beta$-Catenin increased significantly in fat rats induced by high fat. High fat can induce the activation of $\mathrm{Wnt} 3 / \beta$-Catenin and promote the release of inflammatory factors (Gong et al. 2014). Rulifson et al. found in animal experiments that Wnt3a can promote islet B cells (Rulifson et al. 2007). It was found that Wnt5a gene expression was upregulated in obese mice (He et al. 2017). Non-classical Wnt/JNK signaling pathway plays an essential role in cell apoptosis. The activated JNK can further enhance c-jun and other transcription factors in the nucleus, and promote apoptosis (Xie et al. 2011). In this experiment, Wnt5a level of obese children was significantly higher than that of healthy children. After Wnt5a upregulation, JNK expression was significantly upregulated, but the Bcl-2 expression was significantly downregulated. All these indicate that Wnt5a can promote HUVEC apoptosis.

Caveolin-1 exists in many kinds of cells, which can activate many signal pathways to participate in the inflammatory response and regulate inflammatory cells (Lin et al. 2018). Joo et al. (2017) showed that caveolin-1 could mediate the JNK/FOXO3a pathway to induce apoptosis of A549 lung cancer cells. We found that the expression of caveolin-1 was also significantly increased in Wnt5a upregulation, and in the following experiments, 
A
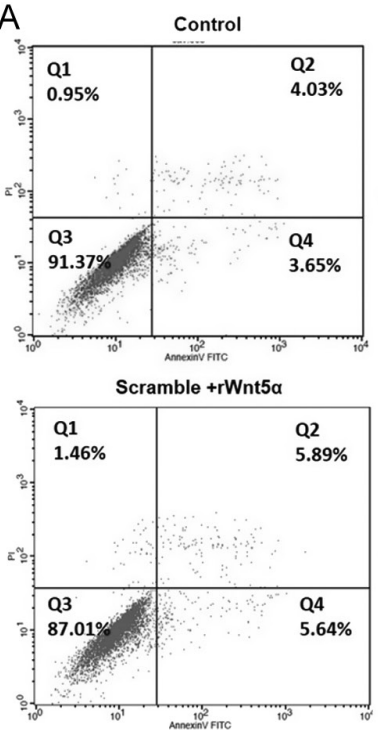
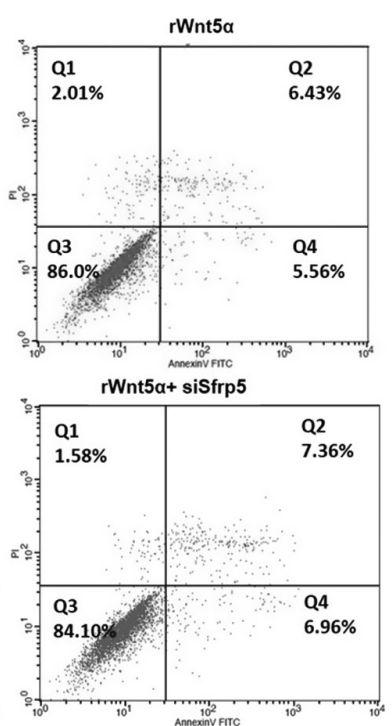
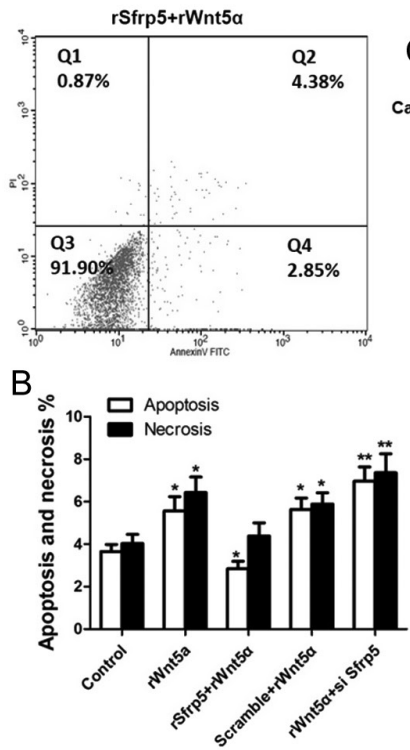

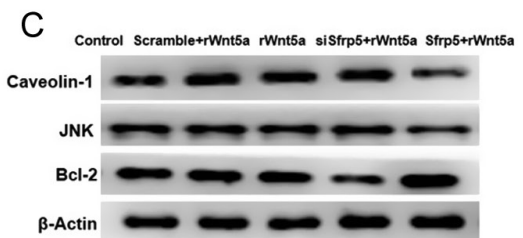

D

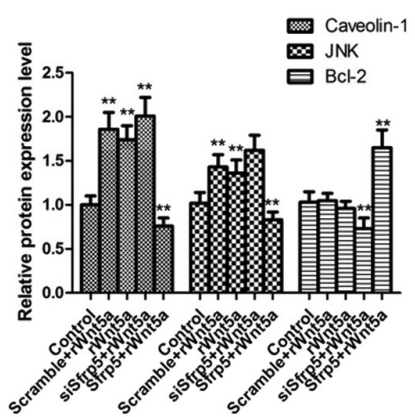

Figure 5

SFRP5 participates in the regulation of HUVEC apoptosis by antagonizing Wnt5a. (A) Flow cytometry detection of cell apoptosis in different groups treated with $100 \mu \mathrm{g} / \mathrm{mL}$ ox-LDL. HUVECs treated with $100 \mu \mathrm{g} / \mathrm{mL}$ ox-LDL for $48 \mathrm{~h}$ were set up with Wnt5a upregulation group and Wnt5a downregulation group, and apoptosis was detected by flow cytometry. The lower-left quadrant of each image represents normal cells, and PI and AV staining were negative (AV-/pl-). The lower right quadrant represents apoptotic cells. AV positive and PI negative (AV+/PI-). The upper right quadrant shows necrotic cells and positive for AV and PI staining (AV+/PI+). (B) Comparison of cell necrosis rate and apoptosis rate in each group. (C and D) Western detection of Caveolin-1, JNK and bcl-2 protein expression in each group $(n=3)$. Compared with the control group, the expression of Caveolin-1 and JNK in rSFRP5 + rwnt5a group decreased significantly, and $\mathrm{Bcl}-2$ increased significantly. ${ }^{*} P<0.05, * \star p<0.01$.

caveolin-1 could mediate the JNK signaling pathway to promote apoptosis.

The biological function of SFRP5 is mainly realized by the Wnt signaling pathway. It can inhibit the activation of Wnt ligands by inhibiting FZ receptors on the surface of cell membrane through competition and playing a role in embryo development, tissue differentiation and metabolism regulation (Prats-Puig et al. 2014, Schulte et al. 2012). Van Camp et al. (2014) studied the polymorphism of SFRP5 gene in obese patients. The results showed that SFRP5 is an important factor regulating adipose tissue development in obese adult men. Tan et al. (2014) found that the serum SFRP5 level in children with obesity was significantly lower than that in children with healthy average weight at the same age, especially in children with obesity and metabolic syndrome. Ouchi et al. (2010) found that SFRP5 is a fat anti-inflammatory factor, and its expression is disturbed in obesity and type 2 diabetes. In obesity, SFRP5 secreted by fat cells plays a beneficial role in metabolic dysfunction by controlling inflammatory cells in adipose tissue. Nakamura et al. (2014) pointed out that in obese patients, due to the lack of SFRP5 expression, Wnt5a over-activated the non-classical Wnt signaling pathway and induced the occurrence of endothelial inflammation in the new museum, which further led to the occurrence of cardiovascular disease. After our study, we found that after upregulating SFRP5, the expression of JNK and Cav-1 decreased significantly. In contrast the expression of Bcl-2 increased significantly, indicating that SFRP5 can resist Wnt5a signaling pathway and inhibit cell apoptosis.

\section{Conclusion}

In this experiment, we found that Wnt5a can promote HUVEC cells' apoptosis through the regulation of caveolin-1 and JNK, but SFRP5 can antagonize this pathway and inhibit the apoptosis. This signal pathway is relatively systematic, which can provide a new treatment for obesity and related diseases.

\section{Declaration of interest}

The authors declare that there is no conflict of interest that could be perceived as prejudicing the impartiality of the research reported. 


\section{Funding}

This study was supported by National Science Foundation of China (No. 81803262) and Science, Technology Planning Project of Xi'an Health Committee (No. J201702018) and Xi'an Science and Technology Project (No. 20YXYJ006(6)).

\section{References}

Ackers I, Szymanski C, Duckett KJ, Consitt LA, Silver MJ \& Malgor R 2018 Blocking Wnt5a signaling decreases CD36 expression and foam cell formation in atherosclerosis. Cardiovascular Pathology 34 1-8. (https:// doi.org/10.1016/j.carpath.2018.01.008)

Akash MSH, Shen Q, Rehman K \& Chen S 2012 Interleukin-1 receptor antagonist: a new therapy for type 2 diabetes mellitus. Journal of Pharmaceutical Sciences 101 1647-1658. (https://doi.org/10.1002/ jps.23057)

Arita Y 2012 Reprint of 'paradoxical decrease of an adipose-specific protein, adiponectin, in obesity'. Biochemical and Biophysical Research Communications 425 560-564. (https://doi.org/10.1016/j. bbrc.2012.08.024)

Bilkovski R, Schulte DM, Oberhauser F, Mauer J, Hampel B, Gutschow C, Krone W \& Laudes M 2011 Adipose tissue macrophages inhibit adipogenesis of mesenchymal precursor cells via wnt- $5 \mathrm{a}$ in humans. International Journal of Obesity 35 1450-1454. (https://doi. org/10.1038/ijo.2011.6)

Danaei G, Ding EL, Mozaffarian D, Taylor B, Rehm J, Murray CJL \& Ezzati M 2009 The preventable causes of death in the United States: comparative risk assessment of dietary, lifestyle, and metabolic risk factors. PLoS Medicine 6 e1000058. (https://doi.org/10.1371/journal.pmed.1000058)

Gong M, Liu C, Zhang L, Zhang H \& Pan J 2014 Loss of the TNFo function inhibits Wnt/ $\beta$-catenin signaling, exacerbates obesity development in adolescent spontaneous obese mice. Molecular and Cellular Biochemistry 391 59-66. (https://doi.org/10.1007/s11010-014-1987-5)

He H, Liang LB, Xia W, Wang XX, AN \& Z-M 2017 Preliminary study on the Wnt5a expression in obese mice with hepatocellular carcinoma. Journal of Sichuan University 48 12-16.

Hotamisligil GS 2003 Inflammatory pathways and insulin action. International Journal of Obesity and Related Metabolic Disorders 27 (Supplement 3) S53-S55. (https://doi.org/10.1038/sj.ijo.0802502)

Hu G, ye RD, Dinauer MC, Malik AB \& Minshall RD 2008 Neutrophil caveolin-1 expression contributes to mechanism of lung inflammation and injury. American Journal of Physiology: Lung Cellular and Molecular Physiology 294 L178-L186. (https://doi.org/10.1152/ ajplung.00263.2007)

Joo JC, Hwang JH, Jo E, Kim YR, Kim DJ, Lee KB, Park SJ \& Jang IS 2017 Cordycepin induces apoptosis by caveolin-1-mediated JNK regulation of Foxo3a in human lung adenocarcinoma. Oncotarget 8 12211-12224. (https://doi.org/10.18632/oncotarget.14661)

Khosravi R, Sodek KL, Xu WP, Bais MV, Saxena D, Faibish M \& Trackman PC 2014 A novel function for lysyl oxidase in pluripotent mesenchymal cell proliferation and relevance to inflammation-associated osteopenia. PLoS ONE 9 e100669. (https://doi.org/10.1371/journal.pone.0100669)

Kikuchi A, Yamamoto H, Sato A \& Matsumoto S 2012 Wnt5a: its signalling, functions and implication in diseases. Acta Physiologica 204 17-33. (https://doi.org/10.1111/j.1748-1716.2011.02294.x)

Lin HK, Lin HH, Chiou YW, Wu CL, Chiu WT \& Tang MJ 2018 Caveolin-1 down-regulation is required for Wnt5a-frizzled 2 signalling in Ha-RasV12-induced cell transformation. Journal of Cellular and Molecular Medicine 22 2631-2643. (https://doi.org/10.1111/jcmm.13531)
Liu J \& Lin A 2005 Role of JNK activation in apoptosis: a double-edged sword. Cell Research 15 36-42. (https://doi.org/10.1038/sj.cr.7290262)

Loos RJ, Lindgren CM, Li S, Wheeler E, Zhao JH, Prokopenko I, Hua J, Inouye M, Freathy R \& Attwood A 2008 Association studies involving over 90,000 people demonstrate that common variants near to MC4R influence fat mass, weight and risk of obesity. Nature Genetics $\mathbf{4 0}$ 768-775. (https://doi.org/10.1038/ng.140)

Ma B, Fey M, Hottiger MO 2015 WNT/ $\beta$-catenin signaling inhibits CBP-mediated RelA acetylation and expression of proinflammatory NF-кB target genes. Journal of Cell Science 128 2430-2436. (https://doi. org/10.1242/jcs.168542)

Nakamura K, Fuster JJ \& Walsh K 2014 Adipokines: a link between obesity and cardiovascular disease. Journal of Cardiology 63 250-259. (https:// doi.org/10.1016/j.jjcc.2013.11.006)

Okamura H, Yang D, Yoshida K, teramachi J \& Haneji T 2014 Reduction of PP2A C $\alpha$ stimulates adipogenesis by regulating the Wnt/GSK-3 $\beta / \beta$ catenin pathway and PPAR $\gamma$ expression. Biochimica et Biophysica Acta 1843 2376-2384. (https://doi.org/10.1016/j.bbamcr.2014.06.008)

Ouchi N, Higuchi A, Ohashi K, Oshima Y, Gokce N, Shibata R, Akasaki Y, Shimono A \& Walsh K 2010 Sfrp5 is an anti-inflammatory adipokine that modulates metabolic dysfunction in obesity. Science 329 454-457. (https://doi.org/10.1126/science.1188280)

Prats-Puig A, Soriano-Rodríguez P, Carreras-Badosa G, Riera-Pérez E, Ros-Miquel M, Gomila-Borja A, de Zegher F, Ibáñez L, Bassols J \& López-Bermejo A 2014 Balanced duo of anti-inflammatory SFRP5 and proinflammatory WNT5A in children. Pediatric Research 75 793-797. (https://doi.org/10.1038/pr.2014.29)

Qin L, Hu R, Zhu N, Yao HL, Lei XY, Li SX, Liao DF \& Zheng XL 2014 The novel role and underlying mechanism of Wnt5a in regulating cellular cholesterol accumulation. Clinical and Experimental Pharmacology and Physiology 41 671-678. (https://doi.org/10.1111/1440-1681.12258)

Rothberg KG, Heuser JE, Donzell WC, Ying YS, Glenney JR \& Anderson RGW 1992 Caveolin, a protein component of caveolae membrane coats. Cell 68 673-682. (https://doi.org/10.1016/00928674(92)90143-z)

Rulifson IC, Karnik SK, Heiser PW, ten Berge D, Chen H, Gu X, Taketo MM, Nusse R, Hebrok M \& Kim SK 2007 Wnt signaling regulates pancreatic $\beta$ cell proliferation. PNAS 104 6247-6252. (https://doi.org/10.1073/pnas.0701509104)

Schulte DM, Müller N, Neumann K, Oberhäuser F, Faust M, Güdelhöfer H, Brandt B, Krone W \& Laudes M 2012 Pro-inflammatory wnt5a and anti-inflammatory sFRP5 are differentially regulated by nutritional factors in obese human subjects. PLOS ONE 7 e32437. (https://doi.org/10.1371/journal.pone.0032437)

Tan X, Wang X, Chu H, Liu H, Yi X \& Xiao Y 2014 SFRP5 correlates with obesity and metabolic syndrome and increases after weight loss in children. Clinical Endocrinology 81 363-369. (https://doi.org/10.1111/ cen.12361)

Van Camp JK, Beckers S, Zegers D, Verrijken A, Van Gaal LF, Van Hul W 2014 Common genetic variation in sFRP5 is associated with fat distribution in men. Endocrine 46 477-484. (https://doi.org/10.1007/ s12020-013-0088-7)

Wang D, Zhang Y \& Shen C 2020 Research update on the association between SFRP5, an anti-inflammatory adipokine, with obesity, type 2 diabetes mellitus and coronary heart disease. Journal of Cellular and Molecular Medicine 24 2730-2735. (https://doi.org/10.1111/ jcmm.15023)

Xie CM, Chan WY, Yu S, Zhao J \& Cheng CHK 2011 Bufalin induces autophagy-mediated cell death in human colon cancer cells through reactive oxygen species generation and JNK activation. Free Radical Biology and Medicine 51 1365-1375. (https://doi.org/10.1016/j. freeradbiomed.2011.06.016)

Received in final form 10 September 2020

Accepted 22 September 2020

Accepted Manuscript published online 23 September 2020 https://joe.bioscientifica.com https://doi.org/10.1530/JOE-20-0328 (c) 2020 Society for Endocrinology Published by Bioscientifica Ltd. Printed in Great Britain 